\title{
ASSOCIAÇÃO ENTRE O NÍVEL DE ATIVIDADE FÍSICA \\ E A ÁREA DE GORDURA VISCERAL EM \\ MULHERES PÓS-MENOPÁUSICAS
}

\author{
ASSOCIATION BETWEEN THE LEVEL OF PHYSICAL ACTIVITY AND AREA OF \\ VISCERAL FAT IN POSTMENOPAUSAL WOMEN
}

\author{
ASOCIACIÓN ENTRE EL NIVEL DE ACTIVIDAD FÍSICA Y EL ÁREA DE GRASA \\ VISCERAL EN MUJERES POSTMENOPÁUSICAS
}

\begin{abstract}
Cristiano Penas Seara Pitanga' (Educador Físico)

Francisco José Gondim Pitanga² (Educador Físico)

Ronaldo Eugénio Calçada Dias Gabriel ${ }^{3}$ (Educador Físico)

Maria Helena Rodrigues Moreira ${ }^{4}$ (Educadora Física)

1. Universidade do Estado da Bahia (UNEB- EAD) e Laboratório Baiano de Ciências do Esporte (SPORTCLIN) Salvador BA-Brasil.

2. Universidade Federal da Bahia (UFBA), Salvador, BA, Brasil.

3. Universidade de Trás-os-Montes e Alto Douro (UTAD), Departamento de Ciências do Desporto, Exercício e Saúde, Centro de Investigação e Tecnologias Agroambientais e Biológicas (CITAB), Vila Real, Portugal. 4. Universidade de Trás-os-Montes e Alto Douro (UTAD), Departamento de Ciências do Desporto, Exercício e Saúde, Centro de Investigação em Desporto, Saúde e Desenvolvimento Humano (CIDESD), Vila Real, Portugal.
\end{abstract}

\section{Correspondência:}

Rua Rodolfo Coelho Cavalcante, 196, Jardim Armação, 41750166, Salvador, BA, Brasil. cpspitanga@gmail.com

\section{RESUMO}

Introdução: O aumento da gordura visceral abdominal está associado à redução dos níveis de atividade física habitual e gera na mulher pós-menopáusica, o surgimento da doença da inatividade física, caracterizada pela manifestação de um conjunto de patologias, entre as quais se incluem diabetes mellitus do tipo 2 e doenças cardiovasculares. Por outro lado, a atividade física regular parece ter impacto significativo na prevenção tanto da gordura intra-abdominal quanto das complicações metabólicas e cardiovasculares. Objetivo: Verificar a associação entre a caminhada e a atividade física de moderada intensidade com a área de gordura intra-abdominal em mulheres pós-menopáusicas. Métodos: A amostra foi composta por 239 mulheres com média de idade de 57,4 \pm 6,6 anos participantes do programa "Menopausa em Forma". Para análise da atividade física foi utilizado o IPAQ versão longa. Consideraram-se os valores de $1.601 \mathrm{kcal} / \mathrm{semana}$ na caminhada e $2.283 \mathrm{kcal} / \mathrm{semana} \mathrm{na}$ atividade física de moderada intensidade como suficientes para prevenção do excesso de gordura intra-abdominal. Utilizou-se a análise de regressão logística para estimar a odds ratio (OR), com intervalo de confiança de 95\%. Resultados: Após análise multivariada observou-se que a caminhada ofereceu proteção contra o excesso de gordura intra-abdominal, principalmente nas mulheres com períodos menstruais regulares, $\mathrm{OR}=0,22(0,08$ $0,62)$ e que não fazem reposição hormonal, $O R=0,05(0,01-0,49)$. Já o nível de atividade física de intensidade moderada ofereceu proteção contra a gordura intra-abdominal independentemente da reposição hormonal ou dos períodos menstruais regulares, $\mathrm{OR}=0,37(0,14-0,66)$. Conclusão: A caminhada e principalmente o nível de atividade física de moderada intensidade podem ser importantes para prevenção do excesso de gordura intra-abdominal e suas complicações metabólicas e cardiovasculares em mulheres pós-menopaúsicas.

Palavras-chave: obesidade abdominal, exercício/fisiologia, atividade motora/fisiologia, pós-menopausa.

\section{ABSTRACT}

Introduction: Increased abdominal visceral fat is associated with reduced levels of physical activity and generates in postmenopausal women the emergence of physical inactivity disease, characterized by a set of conditions including diabetes mellitus type 2 and, cardiovascular disease.. On the other hand regular physical activity appears to have significant impact in preventing both intra-abdominal fat and metabolic and cardiovascular complications. Objective: To investigate the association between walking and physical activity of moderate intensity with the emergence of an area of intra-abdominal fat in postmenopausal women. Methods: The sample consisted of 239 women with a mean age of $57.4 \pm 6.6$ years participants in the "Menopausa em Forma" (fitness in menopause) program. For analysis of physical activity long-version IPAQ was used. The values of 1,601 kcal/week in walking and 2,283 kcal/week in physical activity of moderate intensity were considered sufficient to prevent excessive intra-abdominal fat. We used a logistic regression analysis to estimate the odds ratio (OR) with a confidence interval of 95\%. Results: Multivariate analysis revealed that the walk offered protection against excessive intra-abdominal fat, especially in women with regular menstrual periods, $O R=0.22(0.08-0.62)$ and and in those not receiving hormone replacement therapy, $O R=0.05$ (0.01-0.49). The level of moderate-intensity physical activity offered protection against intra-abdominal fat regardless of hormone replacement or regular periods, $O R=0.37$ (0.14-0.66). Conclusion: We conclude therefore that walking and especially the physical activity of moderate intensity may be important for prevention of excessive intra-abdominal fat and its metabolic and cardiovascular complications in postmenopausal women.

Keywords: obesity, abdominal, exercise/physiology, motor activity/physiology, postmenopause.

\section{RESUMEN}

Introducción: El aumento de la grasa visceral abdominal está asociado a la reducción de los niveles de actividad física habitual y genera en la mujer postmenopáusica el surgimiento de la enfermedad de la inactividad física, caracterizada por la manifestación de un conjunto de patologías, entre las cuales se incluyen diabetes mellitus del 
tipo 2 y enfermedades cardiovasculares. Por otro lado, la actividad física regular parece tener impacto significativo en la prevención tanto de la grasa intraabdominal como de las complicaciones metabólicas y cardiovasculares. Objetivo: Verificar la asociación entre la caminata y la actividad física de moderada intensidad con el área de grasa intraabdominal en mujeres postmenopáusicas. Métodos: La muestra fue compuesta por 239 mujeres con promedio de edad de 57,4 \pm 6,6 años participantes del programa "Menopausia en Forma". Para análisis de la actividad física fue utilizado el IPAQ versión larga. Se consideraron los valores de $1.601 \mathrm{kcal} / \mathrm{semana} \mathrm{en} \mathrm{la} \mathrm{caminata} \mathrm{y} 2.283 \mathrm{kcal} /$ semana en la actividad física de moderada intensidad como suficientes para prevención del exceso de grasa intraabdominal. Se usó el análisis de regresión logística para estimar la odds ratio (OR), con intervalo de confianza de 95\%. Resultados: Después del análisis multivariado se observó que la caminata ofreció protección contra el exceso de grasa intraabdominal, principalmente en las mujeres con períodos menstruales regulares, $O R=0,22(0,08-0,62)$ y que no hacen reposición hormonal, $O R=0,05$ (0,01-0,49). Ya el nivel de actividad física de intensidad moderada ofreció protección contra la grasa intraabdominal independiente de la reposición hormonal o de los períodos menstruales regulares, $O R=0,37$ (0,14-0,66). Conclusión: Se concluye, por lo tanto, que la caminata y principalmente el nivel de actividad física de moderada intensidad pueden ser importantes para prevención del exceso de grasa intraabdominal y sus complicaciones metabólicas y cardiovasculares en mujeres postmenopáusicas.

Palabras clave: obesidad abdominal, ejercicio/fisiología, actividad motriz/fisiología, postmenopausia.

\section{INTRODUÇÃO}

O aumento da gordura visceral abdominal está associado à redução dos níveis de atividade física habitual e gera na mulher pós-menopáusica o surgimento de doença da inatividade física, caracterizada pela manifestação de um conjunto de patologias entre as quais se incluem a diabetes mellitus tipo 2, as doenças cardiovasculares, a depressão, a demência, algumas formas de câncer e a doença de Alzheimer ${ }^{1}$. Por outro lado, a atividade física está associada à diminuição dos níveis de gordura visceral ${ }^{2}$ pressão arterial, glicemia e outros agravos metabólicos e cardiovasculares. Os mecanismos responsáveis pela redução da gordura visceral em função da atividade física incluem, entre outros, a maior resposta lipolítica em função dos efeitos das catecolaminas que provocam acentuada lipólise no tecido abdominal. Por essa razão, a mobilização da gordura durante a atividade física é bastante acentuada, principalmente nos depósitos abdominais ${ }^{3}$. Em recente pesquisa realizada ${ }^{4}$ com o objetivo de identificar a melhor intensidade e duração da atividade física para discriminar a ausência de comorbidades cardiovasculares em mulheres obesas (atividade física analisada em diferentes intensidades) foi observado que níveis de intensidade moderada e com uma duração de 150 minutos por semana estavam relacionados com uma menor incidência de patologias crônico degenerativas nesta população. Estes resultados estão em sintonia com as orientações de exercício procedidas do American College of Sport Medicine para a obesidade e a síndrome metabólica 5 .

Outra recente investigação demonstrou que a prática da atividade física de intensidade leve a moderada, com um gasto calórico entre 1601 e 2283 kcal/semana previne o excesso de adiposidade visceral em mulheres pós-menopáusicas, porém neste estudo não foi testada a associação entre atividade física e gordura visceral com análise de possíveis confundidores ou modificadores de efeito ${ }^{6}$. Visando oferecer uma maior proteção de doenças metabólicas e cardiovasculares e também contrapor o declínio de estrogênio, algumas condutas terapêuticas principalmente à reposição hormonal são aplicadas as mulheres na menopausa, sendo os dados ainda conflitantes em relação aos efeitos da administração de estrogênio. Alguns trabalhos demonstram que a depender do esquema de terapia hormonal administrado pode ocorrer redução na sensibilidade a insulina e aumento no risco do desenvolvimento de comorbidades cardiovasculares ${ }^{7}$. Pode-se também evidenciar que o tempo de menopausa apresenta associação com os fatores de riscos cardiovasculares, incluindo o excesso de gordura abdominal, sendo evidenciado que quanto maior o tempo de menopausa maior o risco de desenvolvimento de resistência insulínica e maiores concentrações de gordura intra-abdominal.

Visando contribuir para a adoção de ações no campo da saúde publica e de intervenções na prática clinica que venham a contribuir para prevenção e tratamento de doenças metabólicas e cardiovasculares em mulheres pós-menopáusicas este estudo pretende examinar a associação do nível de atividade física necessário para proteção do excesso de gordura visceral nesta fase do climatério.

\section{MÉTODOS}

O estudo integra o projeto "Menopausa em Forma", aprovado pela Fundação para a Ciência e Tecnologia (POCI/DES/59049/2004)8 e pela Universidade de Trás-os-Montes e Alto Douro (Portugal), estando orientado para a análise da influência do exercíciono risco cardiovascular e na aptidão física e funcional de mulheres pós-menopáusicas ${ }^{9}$. O recrutamento da amostra foi realizado entre novembro de 2005 e março de 2006 e a inclusão no estudo foi precedida da avaliação da história clinica e reprodutiva.

O estudo atendeu aos procedimentos da Declaração de Helsinki ${ }^{10}$ tendo sido obtido de todas as participantes o consentimento informado assinado. As avaliações foram conduzidas no Laboratório de Aptidão Física, Exercício e Saúde (LAFES) da UTAD, Portugal, por dois técnicos treinados e supervisionados pela investigadora responsável do projeto.

A amostra incluiu 239 mulheres pós-menopáusicas com média de idade de 57,4 \pm 6,6 anos. Os critérios de inclusão observados foram os seguintes: (a) ausência de menopausa precoce; (b) inexistência de significativa doença renal, hepática ou hematológica; (c) inexistência de sintomas de angina de peito ou de enfarte do miocárdio nos últimos 3 meses; (d) não utilização de medicação anti-hipertensiva ou antiarrítmica; (e) ausência de uma hipertensão descontrolada (pressão arterial sistólica $\geq 200$ mmHg e/ou pressão arterial diastólica $\geq 105$ mmHg); (f) não utilização de medicação $\beta$-bloqueadora ou antiarrítmica e; (g) inexistência de condições musculoesqueléticas passíveis de condicionarem a prática de exercício ou serem exarcerbadas pelo mesmo. 0 estudo envolveu a análise da área de gordura visceral $\left(A G V, \mathrm{~cm}^{2}\right)$ e do nível de atividade física em gasto calórico (AF, kcal/semana) nas intensidades caminhada e moderada. As co-variáveis incluíram a regularidade dos períodos menstruais (ciclos menstruais consistentes com uma duração entre 21 e 35 dias), a utilização de métodos contraceptivos, 
o uso de terapia hormonal, o tempo de menopausa, a natureza da menopausa e a idade.

A medição da altura foi efetuada com o estadiometro SECA 220 (Seca Corporation, Hamburg Germany), sendo cumpridos os procedimentos definidos por Heywarde Wagner ${ }^{11}$ e considerado um limite de tolerância de $2 \mathrm{~mm}$. O peso e a AGV foram avaliados com a bioimpedância Octopolar InBody720 (Biospace Co. Ltd, Seoul, Coreia), respeitando os procedimentos referidos no manual do equipamento e as normas de preparação indicadas na literatura. Este equipamento emprega oito eléctrodos (2 posicionados sobre a palma da mão e do polegar e, os outros dois, no calcanhar e na parte anterior do pé) possibilitando a análise da impedância de 5 regiões do corpo (membros superiores, tronco e membros inferiores), através da medição da resistência a cinco frequências $(1 \mathrm{kHz}, 5 \mathrm{kHz}, 50 \mathrm{kHz}, 250 \mathrm{kHz}, 500 \mathrm{kHz}$, $1000 \mathrm{kHz}$ ) e a reactância a três frequências ( $5 \mathrm{kHz}, 50 \mathrm{kHz}$, e $250 \mathrm{kHz}$ ). O In Body 720 é um equipamento válido na avaliação da composição corporal na meia idade ${ }^{12}$, sendo a AGV definida como uma área de corte transversal na zona abdominal, ao nível da L4-L5 e demonstrando uma elevada correlação com a adiposidade intra-abdominal ( $r$ entre 0,759 e 0,922, $p<0,05$ ) obtida por tomografia computorizada ${ }^{13}$..A presença de uma elevada adiposidade visceral foi considerada para valores de $A G V \geq 100 \mathrm{~cm}^{214}$.

Os erros técnicos do peso, da altura e da AGV foram obtidos, com base em medições em duplicado em 10 mulheres pós-menopáusicas, através da fórmula $E T=\left(d^{2} / 2 n\right)^{0,5}(d$, diferença entre as duas avaliações; $n$, número de elementos da amostra). Os valores calculados foram, respectivamente $0,06 \mathrm{~kg}, 0,09 \mathrm{~m}$ e 0,97 $\mathrm{cm}^{2}$.

A atividade física foi avaliada através da versão longa do International Physical Activity Questionnaire (IPAQ), reunindo questões relacionadas com a frequência e duração das atividades físicas realizadas por mais de dez minutos contínuos durante a última semana e abrangendo 4 domínios de atividade física (trabalho, meio de transporte, doméstico e lazer). A validade e confiabilidade deste questionário estão documentadas na literatura ${ }^{15}$.

Cada um dos domínios da atividade física foi expresso em minutos/ semana por meio da multiplicação da frequência semanal pela duração de cada uma das atividades realizadas. Para o cálculo do gasto energético procedeu-se à multiplicação do valor do dispêndio de energia de acordo com a atividade realizada, considerando-se a sua duração e frequência semanal (tempo em minutos/semana). A conversão dos dados obtidos pelo IPAQ em medida de equivalente metabólico (METs) foi realizada de acordo com a proposta de Heymsfield et al. ${ }^{16} \mathrm{O}$ produto do valor de METs da atividade física pela sua duração e frequência resultou no gasto calórico em METs - minutos/semana e a sua conversão em kcal/semana foi obtida através da fórmula: METs-minutos/semana $\times$ (Peso(kg)/60. O questionário foi aplicado e analisado pelo mesmo técnico e obedeceu às orientações de processamento e de análise dos dados do IPAQ, disponíveis no site www.ipaq.ki.se.

\section{Análise Estatística}

Os dados foram analisados recorrendo ao programa estatístico STATA (versão 7.0. Stata Corp., CollegeStation, USA). No primeiro momento, foi realizada a estratificação para verificação da modificação de efeito e confundimento. A análise para modificação de efeito foi executada através da observação das medidas pontuais estrato-específicas e dos seus intervalos de confiança. Quando a medida pontual de um fator, em determinado estrato específico, não estava contida no intervalo de confiança do outro fator no mesmo estrato, essa situação acusava uma modificação do efeito. Foi considerado intervalo de confiança de 95\% pelo método de Mantel-Haenzel. A análise para confundimento foi executada comparando-se a odds ratio (OR) entre a associação bruta e a ajustada pelos possíveis confundidores, sendo considerado o valor de 10\% como parâmetro de identificação da diferença entre as referidas associações.

A análise por meio de regressão estatística foi realizada através do método regressivo, partindo-se do modelo completo e retirando-se uma a uma as possíveis variáveis de confundimento, que, quando suprimidas do modelo, poderiam causar alteração igual ou superior a 10\% na medida pontual de associação entre a atividade física e a área de gordura visceral ${ }^{17}$. Por último, foi estimada a OR (Odds ratio) por meio do modelo que melhor explicava a associação.

As co-variáveis analisadas como possíveis modificadoras de efeito e confundidoras foram às seguintes: idade (variável contínua); tempo de menopausa ( 0 , até 9 anos; 1 , há 10 ou mais anos), terapia hormonal (0, não; 1, sim), natureza da menopausa (0, menopausa natural; 1, menopausa induzida), regularidade da menstruação durante o período fértil (0, não; 1 , sim), utilização de métodos contraceptivos ( 0 , não; 1 , sim).

No processo de modelagem, a idade e o tempo de menopausa foram reconhecidos como confundidores, tanto na associação da caminhada quanto da atividade física moderada com AGV. Na análise da modificação de efeito, as variáveis "regularidade da menstruação durante o período fértil" e "terapia hormonal" foram consideradas modificadores de efeito apenas na associação entre a caminhada e a AGV. Não houve modificadores de efeito na associação entre atividade física moderada e AGV. Diante deste contexto, o melhor modelo para analisar as associações propostas foi o ajustado por idade e tempo de menopausa para a atividade física moderada; e ajustado prioridade e tempo de menopausa e estratificado por períodos menstruais regulares e terapia hormonal para caminhada.

Para efeito do presente estudo a atividade física foi categorizada conforme pontos de corte estabelecidos em recente publicação sobre quantidade de atividade física necessária para prevenção do excesso de gordura visceral em mulheres pós-menopáusicas9: Caminhada: < $1.601 \mathrm{kcal} / \mathrm{sem}=0$; e $>=1,601=1$; Atividade física moderada: $<2.283$ $\mathrm{kcal} / \mathrm{sem}=0 ; \mathrm{e}>=2.283 \mathrm{kcal} / \mathrm{sem}=1$.

\section{RESULTADOS}

A idade média da amostra foi de 57,2 (6,6) anos, revelando a maioria dos seus elementos uma menopausa natural (75,2\%), um tempo de menopausa $\leq 10$ anos $(59,2 \%)$ e o uso de terapia hormonal (tabela 1). A utilização de métodos contraceptivos e a regularidade de períodos menstruais foram documentadas, respectivamente, por 71,6\% e 85\% das mulheres. $O$ gasto energético semanal em atividade física foi de $5411,5 \mathrm{kcal} / \mathrm{semana}$, sendo obtido, sobretudo, com a prática de atividade física de intensidade moderada (3134,8 \pm 4213,8kcal/semana). A AGV variou entre 42,2 e 206,1 cm², apresentando 93,9\% das mulheres níveis iguais ou superiores a $100 \mathrm{~cm}^{2}$.

A associação da atividade física (caminhada e moderada) com a AGV é ilustrada na tabela 2, tendo sido efetuado o ajustamento para a idade e tempo de menopausa. Os resultados demonstram que a presença de níveis mais elevados de atividade física de caminhada e de atividade moderada estão inversamente associados com a AGV em mulheres pós-menopáusicas.

Na tabela 3 observa-se que, após estratificação, a associação entre caminhada e AGV permanece apenas nas mulheres que documentaram períodos menstruais regulares e a não utilização de terapia hormonal. Não foram registrados modificadores de efeito na associação entre atividade física moderada e a AGV.

\section{DISCUSSÃO}

O estudo revelou uma associação inversa e significativa da AFC e AFM com a adiposidade intra-abdominal, sendo a prevenção do excesso de adiposidade central, para níveis de AFC $\geq 1601$ kcal/semana, 
Tabela 1. Análise descritiva da amostra $(n=239)$.

\begin{tabular}{|c|c|c|}
\hline Variáveis & $\begin{array}{l}\text { Média (DP) ou } \\
\text { percentagem }\end{array}$ & $\begin{array}{c}\text { Amplitude ou } \\
\text { intervalo de confiança } \\
\text { (entre parêntesis) }\end{array}$ \\
\hline Idade (anos) & $57,2(6,6)$ & $40,6-79,6$ \\
\hline Peso $(\mathrm{kg})$ & $69,1(11,3)$ & $45,8-108,7$ \\
\hline Altura (m) & $1,55(5,2)$ & $1,42-1,70$ \\
\hline \multicolumn{3}{|l|}{ Área de gordura visceral (AGV) } \\
\hline AGV $(\mathrm{cm} 2)$ & $134,5 \pm 26,9$ & $42,2-206,1$ \\
\hline$<100 \mathrm{~cm} 2$ & 6,1 & $(3,3-9,7)$ \\
\hline$\geq 100 \mathrm{~cm} 2$ & 93,9 & $(90,2-96,6)$ \\
\hline \multicolumn{3}{|l|}{ Atividade física de caminhada(AFC) } \\
\hline AFC (kcal/semana) & $1724,4 \pm 2549,9$ & $0-15178,1$ \\
\hline AFC < 1601 kcal/semana (\%) & 46,7 & $(40,5-53,2)$ \\
\hline AFC $\geq 1601 \mathrm{kcal} /$ semana (\%) & 53,3 & $(46,8-59,5)$ \\
\hline \multicolumn{3}{|l|}{ Atividade física moderada (AFM) } \\
\hline AFM (kcal/semana) & $3134,8 \pm 4213,8$ & $0-25765,5$ \\
\hline AFM < 2283 kcal/semana (\%) & 40,6 & $(4,6-47,2)$ \\
\hline AFM $\geq 2283 \mathrm{kcal} / \mathrm{semana}(\%)$ & 59,4 & $(53,2-65,7)$ \\
\hline Atividade física vigorosa (kcal/semana) & $552,2 \pm 2467,1$ & $0-22304,8$ \\
\hline Atividade física total (kcal/semana) & $5411,5 \pm 6937,2$ & $0-45591,1$ \\
\hline \multicolumn{3}{|l|}{$\begin{array}{c}\text { Regularidade dos } \\
\text { períodos menstruais (\%) }\end{array}$} \\
\hline Não & 15,0 & $(10,9-20,3)$ \\
\hline Sim & 85,0 & $(80,2-89,4)$ \\
\hline \multicolumn{3}{|l|}{ Uso de métodos contraceptivos (\%) } \\
\hline Não & 28,4 & $(22,9-34,4)$ \\
\hline Sim & 71,6 & $(65,6-77,1)$ \\
\hline \multicolumn{3}{|l|}{ Terapia hormonal (\%) } \\
\hline Não & 45,9 & $(39,7-52,4)$ \\
\hline Sim & 54,1 & $(47,6-60,3)$ \\
\hline \multicolumn{3}{|l|}{ Tempo de menopausa (\%) } \\
\hline$\leq 10$ anos & 59,2 & $(52,8-65,3)$ \\
\hline$>10$ anos & 40,8 & $(34,6-47,2)$ \\
\hline \multicolumn{3}{|l|}{ Natureza da menopausa (\%) } \\
\hline Natural & 75,2 & $(69,3-80,4)$ \\
\hline Induzida & 24,8 & $(19,6-30,6)$ \\
\hline
\end{tabular}

DP-desvio padrão

apenas observada nas mulheres não utilizadoras de terapia hormonal e nas que documentaram a presença de ciclos menstruais de 21-35 dias durante o período fértil.

Modestas reduções do peso obtidas através da prática de exercício originam perdas preferenciais de gordura visceral, preservando a massa magra e prevenindo o declínio do dispêndio energético. Após a perda de peso, a manutenção da gordura visceral exige um dispêndio calórico semanal de pelo menos $2000 \mathrm{kcal}^{18}$, sendo a referida manutenção essencial para a melhoria dos triglicerídios e do colesterol das lipoproteínas de elevada densidade na mulher pós-menopáusica.

Perez e Garbe ${ }^{19}$ sugerem que na menopausa a mulher deve acumular 150 a 300 minutos por semana de atividade moderada ou 100 a 150 minutos de atividade física vigorosa ou obter quantidade equivalente, combinando os dois tipos de intensidade. Os nossos resultados propõem que, independentemente da terapia hormonal e da natureza dos ciclos menstruais na fase reprodutiva, um dispêndio energético semanal igual ou superior a $2283 \mathrm{kcal}$ de atividade moderada está articulado a uma melhor AGV. As pesquisas sugerem também que, nas mulheres não utilizadoras de TH e com períodos menstruais regulares, um dispêndio energético de pelo menos 1601 kcal/semana de
Tabela 2. Associação da caminhada e da atividade física moderada com a área de gordura visceral ajustada para a idade e tempo de menopausa.

\begin{tabular}{c|c|c}
\hline Variáveis & OR & IC (95\%) \\
\hline Atividade física de caminhada (AFC) & & \\
\hline AFC $<1601 \mathrm{kcal} / \mathrm{semana}$ & 1.00 & \\
\hline AFC $\geq 1601 \mathrm{kcal} / \mathrm{semana}$ & 0.29 & $0.11-0.75$ \\
\hline Atividade física moderada (AFM) & & \\
\hline AFM $<2283 \mathrm{kcal} / \mathrm{semana}$ & 1.00 & $0.14-0.66$ \\
\hline AFM $\geq 2283 \mathrm{kcal} / \mathrm{semana}$ & 0.37 & \\
\hline
\end{tabular}

OR, Oddsratio; IC, intervalo de confiança.

Tabela 3. Associação da caminhada com a área de gordura visceral ajustada para a idade e o tempo de menopausa e estratificada por regularidade dos períodos menstruais e terapia hormonal.

\begin{tabular}{c|c|c}
\hline Variáveis & OR & IC (95\%) \\
\hline Regularidade dos períodos menstruais & & \\
\hline Não & 1.14 & $0.83-1.57$ \\
\hline Sim & 0.22 & $0.08-0.62$ \\
\hline Terapia hormonal & & $0.01-0.49$ \\
\hline Não & 0.05 & $0.19-2.07$ \\
\hline Sim & 0.63 &
\end{tabular}

OR, Odds ratio; IC, intervalo de confiança.

atividade física de caminhada (caminhada no trabalho, no tempo de lazer e em deslocamento ativo) previne o excesso de AGV. Esta forma de atividade física moderada e de baixo impacto é facilmente adaptada por mulheres com condições crônicas de saúde, problemas de mobilidade ou limitados níveis de aptidão física, constituindo um meio adequado para a aquisição de níveis recomendados de atividade física.

Nas recentes orientações das três principais sociedades internacionais de menopausa, não é feita referência ao efeito da TH na adiposidade intra-abdominal, sendo contudo focados os seus benefícios ao nível da doença de Alzeimer e, certas formas de cancro, doença coronária e diabetes mellitus, condições acomunadas ao excesso de AGV20. A literatura revela também resultados contraditórios relacionados com a influência da TH na composição corporal da mulher pós-menopáusica, espelhando distintas condições de realização dos estudos, incluindo as doses de estrogénio utilizadas, o momento de iniciação da terapia e os diferenciados produtos (estrogênios, progesterona, terapias combinadas, androgênios ou tibolona) e vias de administração (oral, transdérmica e vaginal) da terapia. Assim, alguns autores ${ }^{21}$ reportam uma atenuação do aumento da adiposidade central na mulher pós-menopáusica com a utilização da TH, enquanto outros apontam efeitos limitados ou inexistentes ${ }^{22,23}$. A European Menopause Society e a International Menopause Society salientam que a indicação da TH na perimenopausa e na pós-menopausa deve fazer parte de um conjunto de estratégias de promoção da saúde da mulher, incluindo o exercício físico, e que a sua prescrição deverá ser individualizada e iniciada antes dos 60 de idade.

A pesquisa revela algumas limitações que nos merecem os seguintes comentários. Em primeiro lugar, os resultados podem não ser generalizados a mulheres pós-menopáusicas de outros grupos raciais/ étnicos e teria sido importante o recrutamento de uma amostra mais representativa. Por outro lado, a apreciação da adiposidade intra-abdominal carece da aplicação de outras metodologias de maior precisão, 
sendo a espectroscopia por ressonância magnética e a imagem por ressonância magnética considerados métodos padrão na avaliação do conteúdo e da distribuição da massa gorda ${ }^{24}$. Futuras investigações que definam detalhes relacionados com a dieta e os níveis de intensidade da atividade física, apreciados por exemplo por acelerometria, podem ajudar a clarificar a associação entre a AF e a adiposidade visceral, melhorando a intervenção dos profissionais que atuam no âmbito da ciências do desporto e conferindo à mulher pós-menopáusica a aquisição de instrumentos que contribuam para manter-se ativa e produtiva do ponto de vista pessoal e social.

\section{CONCLUSÃO}

Os resultados do estudo sugerem que níveis mais elevados de AFC e de AFM estão inversamente associados com a AGV em mulheres pós-menopáusicas. A AFM $22283 \mathrm{kcal} / \mathrm{semana}$ e a AFC $\geq 1601$ kcal/semana (esta última apenas em mulheres com períodos menstruais regulares e sem TH) estão articulados a uma melhor adiposidade intra-abdominal

Todos os autores declararam não haver qualquer potencial conflito de interesses referente a este artigo.

\section{REFERÊNCIAS}

1. Pedersen BK. The diseasome of physical inactivity--and the role of myokines in muscle--fat cross talk. J Physiol. 2009;587(Pt 23):5559-68.

2. Kotani K, Tokunaga K, Fujioka S, Kobatake T, Keno Y, Yoshida S, et al. Sexual dimorphism of age-related changes in whole-body fat distribution in the obese. Int J ObesRelatMetabDisord. 1994;18(4):207-2.

3. Després JP, Pouliot MC, Moorjani S, Nadeau A, Tremblay A, Lupien PJ, et al. Loss of abdominal fat and metabolic response to exercise training in obese women. Am J Physiol. 1991;261(2 Pt 1):E159-67.

4. Pitanga FJG, Lessa I, Pitanga CPS, Costa MC. Atividade física na prevenção das comorbidades cardiovasculares em mulheres obesas: quanto é suficiente?.Rev Bras AtivFísSaúde. 2011;16:334-8.

5. ACSM's guidelines for exercise testing and prescription.8th ed. Philadelphia: Lippincott Williams \& Wilkins; 2010 .

6. Pitanga CPS, Pitanga FJG, Beck CC, Gabriel REC, Moreira MHR. Nível de atividade física para prevenção do excesso de gordura visceral em mulheres pós-menopáusicas: quanto é necessário? Arq Bras EndocrinolMetabol. 2012;:;6(6):358-63.

7. Nelson ME, Rejeski WJ, Blair SN, Duncan PW, Judge JO, King AC, et al. Physical activity and public health in older adults: recommendation from the American College of Sports Medicine and the AmericanHeart Association. Med Sci Sports Exerc. 2007;39(8):1435-45.

8. Moreira H. Menopausa em forma: programa de promoção do exercício e da saúde em mulheres pós-menopáusicas. In: Dantas E. Actividade física e envelhecimento saudável. Rio de Janeiro: Shape; 2008. p. 181-201.

9. Moreira H. Influência do exercício físico no risco cardiovascular e na aptidão física e funcional de mulheres pós-menopáusicas. In: ProjectoRefa POCTI/DES/59049/2004, Fundação para a Ciência e a Tecnologia; 2004.

10. World Medical Association. World medical association declaration of Helsinki: ethical principles for medical research involving human subjects. Seoul, Corea: WMA General Assembly; 2008.

11. Heyward VH, Wagner D. Applied body composition assessment. 2nd ed. Champaign: Human Kinetics; 2004.

12. Ling $\mathrm{CH}$, de Craen AJ, Slagboom PE, Gunn DA, Stokkel MP, Westendorp RG, et al. Accuracy of direct segmental multi-frequency bioimpedance analysis in the assessment of total body and segmental body composition in middle-aged adult population. ClinNutr. 2011;30(5):610-5.
13. Ogawa H, Fujitani K, Tsujinaka T, Imanishi K, Shirakata H, Kantani A, et al. InBody 720 as a new method of evaluating visceral obesity. Hepatogastroenterology. 2011;58(105):42-4.

14. Després JP, Lamarche B. Effects of diet and physical activity on adiposity and body fat distribution: implications for the prevention of cardiovascular disease. Nutr Res Rev. 1993;6(1):137-59.

15. Craig CL, Marshall AL, Sjöström M, Bauman AE, Booth ML, Ainsworth BE, et al. International physica activity questionnaire: 12-country reliability and validity. Med Sci Sports Exerc. 2003;35(8):1381-95.

16. HeymsfieldSI, Lohman T, Wang Z, Going S. Human body composition. Champaingh: Human Kinetics; 2005.

17. Hosmer JR, Lemeshow S. Appled logistic regression. John Wiley \& Sons: New York; 1998.

18. Lovejoy JC, Champagne CM, de Jonge L, Xie H, Smith SR. Increased visceral fat and decreased energy expenditure during the menopausal transition. Int J Obes (Lond). 2008;32(6):949-58.

19. Perez K, Garber C. Exercise prescription for the menopausal years: promoting and enhancing well-being ACSM's HealthFitness J. 2011;15(3):8-14

20. de Villiers TJ, Pines A, Panay N, Gambacciani M, Archer DF, Baber RJ, et al. Updated 2013 Internationa Menopause Society recommendations on menopausal hormone therapy and preventive strategies for midlife health. Climacteric. 2013;16(3):316-37

21. Sites CK, L'Hommedieu GD, Toth MJ, Brochu M, Cooper BC, Fairhurst PA. The effect of hormone replacement therapy on body composition, body fat distribution, and insulin sensitivity in menopausal women: a randomized, double-blind, placebo-controlled trial . J Clin Endocrinol Metab. 2005; 90(5):2701-7

22. Chen Z, Bassford T, Green SB, Cauley JA, Jackson RD, LaCroix AZ, et al. Margolis KL. Postmenopausa hormone therapy and body composition - a substudy of the estrogen plus progestin trial of the Women's Health Initiative. Am J ClinNutr. 2005;82(3):651-6.

23. Gower BA, Muñoz J, Desmond R, Hilario-Hailey T, Jiao X. Changes in intra-abdominal fat in early postmenopausal women: effects of hormone use. Obesity (Silver Spring). 2006;14(6):1046-55.

24. Thomas E, Fitzpatrick J, Malik S, Taylor-Robinson S, Bell J. Whole Body fat: content and distribution progress in nuclear Magnetic Resonance Spectroscopy. 2013;73:56-80 